\title{
Simultaneous Dual-tracer PET Imaging of the Rat Brain and its Application in the Study of Cerebral Ischemia
}

\author{
Francisca P. Figueiras, ${ }^{1}$ Xavier Jiménez, ${ }^{1}$ Deborah Pareto,,${ }^{1,3}$ Vanessa Gómez, ${ }^{2,3}$ \\ Jordi Llop, ${ }^{2,3}$ Raul Herance, ${ }^{1}$ Santiago Rojas, ${ }^{1}$ Juan D. Gispert ${ }^{1,3}$ \\ ${ }^{1}$ Institut d'Alta Tecnologia-Parc de Recerca Biomèdica de Barcelona (PRBB), CRC Corporació Sanitària, Edifici PRBB c/Dr. Aiguader, 88, \\ 08003 Barcelona, Spain \\ ${ }^{2}$ CIC-BiomaGUNE, Unidad de Imagen, San Sebastián, Spain \\ ${ }^{3}$ CIBER en Bioingeniería, Biomateriales y Nanomedicina (CIBER-BBN), Zaragoza, Spain
}

\begin{abstract}
Purpose: This study evaluates the performance of simultaneous dual-tracer technique (SDTT) in static positron emission tomography (PET) studies using 2-deoxy-2- $\left[{ }^{18} \mathrm{~F}\right]$ fluoro-D-glucose and $\left[{ }^{13} \mathrm{~N}\right]$ ammonium as radiotracers.

Procedures: The effects of applying SDTT either to the reconstructed image or directly to the sinogram, different rebinning algorithms, total acquisition time, and frame duration were investigated; first, using a specific phantom and later using an in vivo application of the study of cerebral ischemia.

Results: The best results were obtained using the image method with single-slice rebinning and a total acquisition time of at least $20 \mathrm{~min}$. Frame duration did not affect SDTT performance. The method was also applied in rats with transient cerebral ischemia to simultaneously study cerebral blood flow and cerebral glucose metabolism.

Conclusion: The results encourage the use of SDTT as it has very good potential for examining two different biological processes at the same time utilising rodent PET scanners.
\end{abstract}

Key words: PET radiotracers, Blood flow, Glucose metabolism, Noise, Mathematical analysis

\section{Introduction}

$\mathrm{P}$ ositron emission tomography (PET) and single-photon emission computed tomography (SPECT) are nuclear medicine imaging techniques that produce a three-dimen-

\footnotetext{
Manuscript Category and its Significance: article - to our knowledge, this is the first report of simultaneous dual-isotope PET imaging in vivo, after determining the optimum parameters from phantom experiments. While some other articles study the applicability of this technique from the mathematical perspective or conducted phantom experiments solely, we demonstrate its applicability for the in vivo simultaneous determination of cerebral blood perfusion and glucose consumption.
}

Correspondence to: Juan D. Gispert; e-mail: jgispert@crccorp.es sional image of functional processes in the body. They measure in vivo the biodistribution of radionuclide-labelled imaging agents. SPECT allows for dual-tracer acquisition to image the distribution of two radiotracers in the body simultaneously. In this technique, two tracers labelled with gamma-emitting isotopes with different photon energies are introduced into the patient, and the separation of these two tracers is achieved by energy discrimination [1-3]. Because the two tracers are in the body at the same time and are measured simultaneously, the dual-tracer technique can reduce problems of patient movement, image alignment, and physiological changes.

This technique of energy discrimination is not applicable in PET as the photons emitted from positron annihilation have the same energy $(511 \mathrm{KeV})$. To 
overcome this limitation, tracer discrimination using radioactive decay rates of different positron emitters was originally proposed by Huang et al. [4]. However, this procedure is not routinely applied since it increases the image noise, depending on the relative half-lives of the isotopes and the time duration of the scans as studied in [5].

While dual-tracer PET studies are of clinical interest, they do require two separate scans. For example, a myocardial viability examination requires an assessment of glucose consumption using 2-deoxy-2- $\left[{ }^{18} \mathrm{~F}\right]$ fluoro-D-glucose $\left({ }^{18} \mathrm{~F}\right.$ FDG) and perfusion using $\left[{ }^{13} \mathrm{~N}\right]$ ammonium $\left({ }^{13} \mathrm{~N}-\mathrm{NH}_{4}{ }^{+}\right)$. Simultaneous imaging techniques alleviate the problems relating to image alignment and physiological changes inherent in successive imaging. Other sequential and simultaneous dual-tracer PET scans have been investigated [4-9], such as PET kinetic analysis with dual-tracer injection, where the aim of the work was to investigate the optimal injection intervals and administration dose ratios of the two tracers [6-8].

The simultaneous dual-tracer technique (SDTT) relies on the ability to distinguish each radiotracer on the basis of its unique radioactive decay rate. The technique can be performed at the dynamic sinogram or image levels. This study describes and compares the two different dualtracer techniques: the Sinogram Method (SM) and the Image Method (IM). SDTT was performed on both phantom and in vivo data, using ${ }^{18} \mathrm{~F}-\mathrm{FDG}$ and ${ }^{13} \mathrm{~N}$ $\mathrm{NH}_{4}{ }^{+}$radiotracers with half-life times of 109.77 and $9.97 \mathrm{~min}$, respectively. The first aim of this study was to assess the extent to which the experimental settings could influence the SDTT results. The impact on the SDTT of some frequent experimental settings, such as the rebinning algorithms, the total acquisition time and the frame duration were also investigated individually for both methods. Finally, the primary interest of this work was to test the performance of SDTT using the recommended experimental settings on an in vivo dataset. To this end, SDTT was applied in a rat model of transient cerebral ischemia. In this model of ischemic stroke, a mismatch is found during the first hours after reperfusion between brain glucose metabolism and blood flow [10]. The dual-tracer approach would be very useful in helping to understand the relationship between the various pathophysiologic processes that occur during the acute phase of cerebral ischemia. The injury mechanisms that occur in ischemic damage change rapidly over time and, therefore, it may not be possible to study two different processes in the same animal using two separate single-tracer acquisitions.

\section{Material and Methods}

\section{Theory}

Dynamic PET imaging of two radionuclides with decay constants $\lambda_{\mathrm{A}}$ and $\lambda_{\mathrm{B}}$ with initial $(t=0)$ activity concentrations
$m_{A}$ and $m_{B}$, will yield an observed activity $m$ along $n$ time frames:

$$
\begin{aligned}
m\left(x, y, z, t_{i}\right)= & m_{A}(x, y, z, 0) \cdot e^{-\lambda A \cdot t A} \\
& +m_{B}(x, y, z, 0) \cdot e^{-\lambda B \cdot t B} \\
& (i=1, \ldots, n)
\end{aligned}
$$

where, $m_{A}$ and $m_{B}$ can be estimated using the nonlinear least squares fitting method [4]. In this study, the unknown parameters were estimated using nonlinear least squares data fitting by the Gauss-Newton method, using MATLAB software (The MathWorks Inc., Natick, MA, USA).

The method described above can be applied to dynamic sinograms or directly to the reconstructed dynamic images. This study analysed and compared the two different methods of SDTT. The first method is the SM, which uses the dynamic sinogram of the dual-tracer acquisition as the initial source. Because of the different radioactive decay rates of ${ }^{18} \mathrm{~F}$ and ${ }^{13} \mathrm{~N}$ from the dynamic sinogram and using a nonlinear least squares fitting method, it is possible to determine the two static sinograms that correspond to the ${ }^{18} \mathrm{~F}-\mathrm{FDG}$ and ${ }^{13} \mathrm{~N}_{-} \mathrm{NH}_{4}{ }^{+}$radiotracers. Subsequently, both images were reconstructed and analysed. The second method, the IM, differs from the previous one in that it uses the resulting dynamic image of the dual-tracer PET acquisition as the source, providing as a result two static images that correspond to the two radiotracers, ${ }^{18} \mathrm{~F}-\mathrm{FDG}$ and ${ }^{13} \mathrm{~N}-\mathrm{NH}_{4}{ }^{+}$(Fig. 1).

\section{Phantom Experiments}

To investigate the impact of the usual post-processing parameters in PET imaging, the standard filtered backprojection (FBP) reconstruction algorithm was used and two different rebinning algorithms were applied: single-slice rebinning (SSRB) and Fourier rebinning (FORE).

Rebinning algorithms seek to convert the collected 3D data, a set of parallel transverse sinograms, so they can be reconstructed using conventional 2D FBP methods. The simplest of these methods, SSRB [11], takes the average axial location of a detected event and places the event in the sinogram(s) that most closely corresponds to the axial position. Along the central axis of the scanner, this approximation works perfectly. However, it steadily becomes worse with increasing radial distance. More recently, a technique known as FORE has been introduced [12]. The details of FORE are beyond the scope of this text, but it is based on a principle that relates the 2D Fourier transform of the oblique sinograms to the 2D Fourier transform of the transverse sinograms. Although this is still an approximate method, it yields substantially better results than SSRB, even for large objects and large acceptance angles, while retaining much of the advantage in terms of short reconstruction times. This has become the algorithm of choice for very large 3D datasets, for example, those from dynamic PET studies involving perhaps 20 to 30 frames of 3D data.

SSRB was applied using different values for the span and ring difference to evaluate their impact on the SDTT. This technique was also applied using different total acquisition times $(5,10,20$, and $30 \mathrm{~min}$ ), different frame durations $(10,30,60,120$, and $300 \mathrm{~s}$ ), and therefore a different number of frames, in order to 


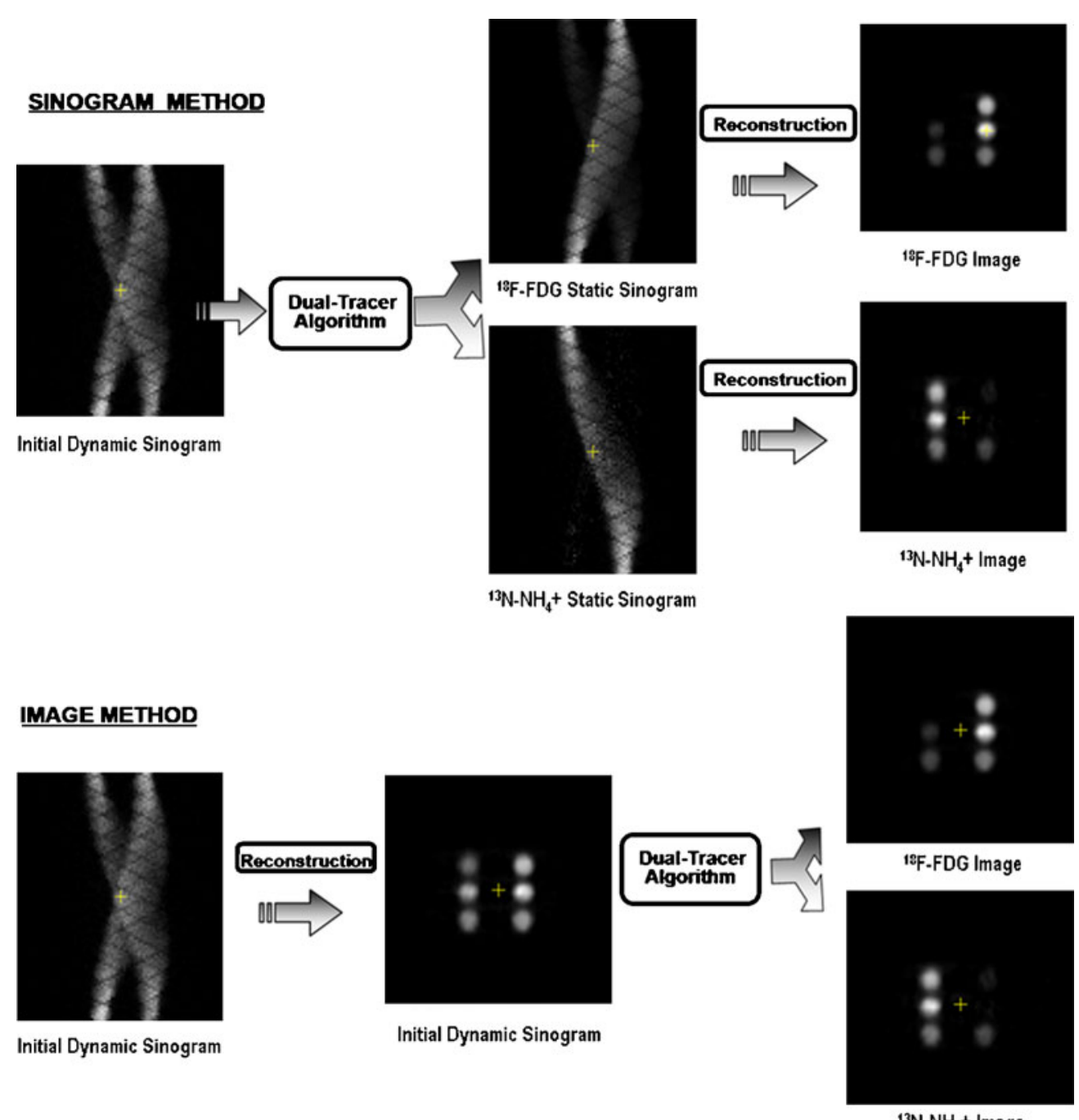

${ }^{13} \mathrm{~N}-\mathrm{NH}_{4}+$ Image

Fig. 1. Scheme of both simultaneous dual-tracer technique methods; the Sinogram Method and the Image Method.

evaluate how these factors influence the results. Each parameter was studied individually and each different rebinning algorithm was applied using different total acquisition times and frame durations.

SDTT was applied to a phantom consisting of six Eppendorf tubes filled with different relative concentrations of ${ }^{18} \mathrm{~F}$-FDG and ${ }^{13} \mathrm{~N}_{-} \mathrm{NH}_{4}{ }^{+}$and imaged for $33 \mathrm{~min}$ in a Siemens micro-PET R4. Because SDTT comprises the different radioactive decay rates of each radiotracer, no decay correction or attenuation was applied. The initial activity concentration of ${ }^{18} \mathrm{~F}-\mathrm{FDG}$ and ${ }^{13} \mathrm{~N}_{-} \mathrm{NH}_{4}{ }^{+}$was $12.58 \mathrm{MBq} / \mathrm{ml}(340 \mu \mathrm{Ci} / \mathrm{cc})$ and $6,475 \mathrm{MBq} / \mathrm{ml}(175 \mu \mathrm{Ci} / \mathrm{cc})$, respectively. The volume ratios of ${ }^{18} \mathrm{~F}-\mathrm{FDG}$ to ${ }^{13} \mathrm{~N}-\mathrm{NH}_{4}{ }^{+}$introduced into each of the Eppendorf tubes were as follows: tube 1, 83.33:16.67; tube 2, 100:0; tube 3, 60:40; tube 4, 40:60; tube 5, 20:80; and tube 6, 0:100.

Sinograms were histogrammed using both SSRB and 3D rebinning with the aid of the tomography software. The dimensions were $84 \times 96$ and 63 planes. The 3D sinograms present 21 segments and the default span and ring difference settings for micro-PET R4 are 3 and 31, respectively. For the SSRB rebinning, the settings on the micro-PET protocol setup page give a ring difference of 15 and a span of 31. In this study, SSRB was applied using two different span and ring difference values: one using the default values of the micro-PET protocol and the other using a span of 3 and a ring difference of 31 .

Scans were reconstructed using the standard FBP with a ramp filter (cut-off frequency $=0.5 \mathrm{~mm}^{-1}$ ), using an image matrix size of $128 \times 128$, giving images consisting of 63 planes with a voxel size of $0.845247 \times 0.845247 \times 1.2115 \mathrm{~mm}^{3}$. Reconstruction was implemented using both SSRB (also using two different span and ring difference values) and FORE. All data were corrected for random coincidences, dead time and normalisation.

\section{Evaluation of Phantom Experiments}

In order to quantitatively evaluate the results of both methods, the accuracy, signal-to-noise ratio (SNR), and coefficient of variation $(\mathrm{CV})$ of their resulting images were measured and compared. Seven regions of interest (ROIs) were drawn, one for each Eppendorf tube and one for the background. The ROI of the background corresponds to the entire image except the six Eppendorf ROIs $\left(\mathrm{ROI}_{\text {background }}=\right.$ Image $\left.-\mathrm{ROI}_{1: 6}\right)$. 
The accuracy of both methods was calculated. The accuracy of SDTT can be measured through the percentage difference between the recovered activity concentration values of each $\mathrm{ROI}$ and the theoretical activity concentrations of ${ }^{18} \mathrm{~F}-\mathrm{FDG}$ and ${ }^{13} \mathrm{~N}_{-} \mathrm{NH}_{4}{ }^{+}$as determined by the initial activity concentrations and volumes at each Eppendorf tube.

The behaviour of the image noise in this process was also analysed. From the mean and standard deviation of each ROI, including the ROI of the background, the SNR was calculated using the hottest regions as reference:

$$
\mathrm{SNR}=\frac{\left(X_{\text {Hot Region }}-X_{\text {Background }}\right)}{\sigma_{\text {Background }}}
$$

where, $X$ is the mean of the ROI and $\sigma$ its standard deviation (9). The hottest regions correspond to the ROI containing either ${ }^{18} \mathrm{~F}$ -

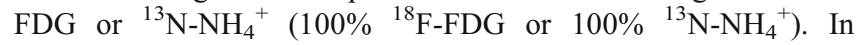
addition, in order to understand the noise level of the image value within the ROIs, the coefficient of variation of each of them was calculated and analysed.

\section{In vivo Experiments}

Kinetic evaluation Before applying SDTT to the in vivo data, it is necessary to have a thorough understanding of how each radiotracer behaves in the rat brain. SDTT assumes that there will be no change in the tracer concentrations during the acquisition. It is therefore critical to ensure that this condition is satisfied when applying SDTT. To this end, two single dynamic acquisitions were performed in two normal male Sprague Dawley rats, one for each radiotracer, ${ }^{18} \mathrm{~F}-\mathrm{FDG}$ and ${ }^{13} \mathrm{~N}$ $\mathrm{NH}_{4}{ }^{+}$. From each individual radiotracer image, a ROI of the brain was drawn and the time-activity curve (TAC) was calculated (Fig. 2).

Another three adult male Sprague Dawley rats (300-400 g body weight) were used for brain ischemia experiments. Animal work was conducted in compliance with Spanish legislation on the "Protection of Animals Used for Experimental and other Scientific Purposes" and in accordance with EU Directives. Transient focal ischemia was induced by intraluminal occlusion of the right middle cerebral artery (MCA), in accordance with the protocol described by Martin el al. [13]. Briefly, the rats were anaesthetized with $4 \%$ isofluorane in $\mathrm{O}_{2}$ and maintained under anaesthesia during surgery with the same gas at $2 \%$. A nylon monofilament with a heat-bonded tip was introduced into the right carotid artery up to junction with the MCA. After occlusion, the anaesthesia was removed and the rats were placed in their cages. Two hours later, the animals were anaesthetized again, the filament was fully removed, and the common carotid artery was released to enable reperfusion.

Three different experiments were carried out to assess the feasibility of the SDTT for determining brain glucose consumption and perfusion in vivo. The first experiment was designed to obtain an abnormal asymmetric ${ }^{18}$ F-FDG image and a normal symmetric ${ }^{13} \mathrm{~N}_{-} \mathrm{NH}_{4}{ }^{+}$image, while the second experiment sought to achieve the opposite. The third experiment was designed to obtain normal symmetric ${ }^{18} \mathrm{~F}-\mathrm{FDG}$ and ${ }^{13} \mathrm{~N}_{-} \mathrm{NH}_{4}{ }^{+}$images (shamoperated experiment).

Experiment 1: Approximately $1 \mathrm{mCi}$ of ${ }^{18} \mathrm{~F}-\mathrm{FDG}$ was injected into the animals $18 \mathrm{~h}$ after the reperfusion procedure. After $1 \mathrm{~h}$ of uptake, $6 \mathrm{mCi}$ of ${ }^{13} \mathrm{~N}$ $\mathrm{NH}_{4}{ }^{+}$were injected, and, after $5 \mathrm{~min}$, dynamic images were acquired for $80 \mathrm{~min}$. Data acquired during the first 30 min were sorted into $303 \mathrm{D}$ sinograms $(\operatorname{span}=3$; ring difference $=31)$ and reconstructed using the FBP algorithm, yielding frames of 1 min duration, which were processed later with the dual-isotope algorithm. The last $20 \mathrm{~min}$ of the acquisition were sorted into a static sinogram and were considered to be free of any remaining ${ }^{13} \mathrm{~N}_{-} \mathrm{NH}_{4}{ }^{+}$because more than $1 \mathrm{~h}$ (about six half-lives) had passed since the injection. This image was then used for comparison with the ${ }^{18}$ F-FDG image resulting from the dualisotope procedure. In this case, it is expected to have a normal symmetric perfusion image since the animals had been reperfused and abnormal asymmetric glucose consumption due to the ischemic injury. After image acquisition, the animal was euthanized and its brain removed. The brain was sectioned axially and the fresh sections obtained were incubated at room temperature with a $1 \%$ solution of 2,3,5-triphenyl tetrazolium chloride in $0.1 \mathrm{M}$ phosphate buffer at $\mathrm{pH} 7.4$ for $10 \mathrm{~min}$. Finally, the sections were fixed with $4 \%$ paraformaldehyde solution and $4 \%$ phosphate buffer. With this method, the viable tissue appears red-coloured while the infarcted regions remain pale.

Experiment 2: Here, $0.5 \mathrm{mCi}$ of ${ }^{18} \mathrm{~F}-\mathrm{FDG}$ was injected into the animal $1 \mathrm{~h}$ before the MCA occlusion procedure, with the expectation of normal ${ }^{18} \mathrm{~F}$-FDG uptake. The MCA was then occluded and $5 \mathrm{mCi}$ of ${ }^{13} \mathrm{~N}$ $\mathrm{NH}_{4}{ }^{+}$were injected $5 \mathrm{~min}$ before the start of acquisition, which was performed using the same protocol as in the previous experiment. In this case, a normal and symmetric ${ }^{18} \mathrm{~F}$-FDG image was expected, together with an abnormal asymmetric ${ }^{13} \mathrm{~N}_{-} \mathrm{NH}_{4}{ }^{+}$image, since the animals were not reperfused.

Experiment 3: In this case, $1 \mathrm{mCi}$ of ${ }^{18} \mathrm{~F}-\mathrm{FDG}$ was injected into the animal before the surgery, again with the expectation of normal ${ }^{18} \mathrm{~F}-\mathrm{FDG}$ uptake. The animal was subjected to the same surgical procedure, but without introduction of the occlusion filament. Immediately after the surgery, $2 \mathrm{mCi}$ of ${ }^{13} \mathrm{~N}_{-} \mathrm{NH}_{4}{ }^{+}$were injected in the sham-operated rat $5 \mathrm{~min}$ before starting the acquisition. Image acquisition was carried out using the same proto$\mathrm{col}$ as in the previous experiments. In this experiment, there was no MCA occlusion and therefore the surgery did not cause blood flow asymmetry. Normal and symmetric ${ }^{18} \mathrm{~F}-\mathrm{FDG}$ and ${ }^{13} \mathrm{~N}_{-} \mathrm{NH}_{4}{ }^{+}$ images were expected.

In order to quantitatively evaluate the performance of SDTT in the in vivo studies, two ROIs were drawn in the resulting ${ }^{18} \mathrm{~F}$ FDG and ${ }^{13} \mathrm{~N}_{-} \mathrm{NH}_{4}{ }^{+}$images: affected and contralateral hemispheres of the brain. The mean of each ROI and the ratio of the affected vs. contralateral hemispheres was calculated and analysed. 
[18F]FDG Time-Activity Curve

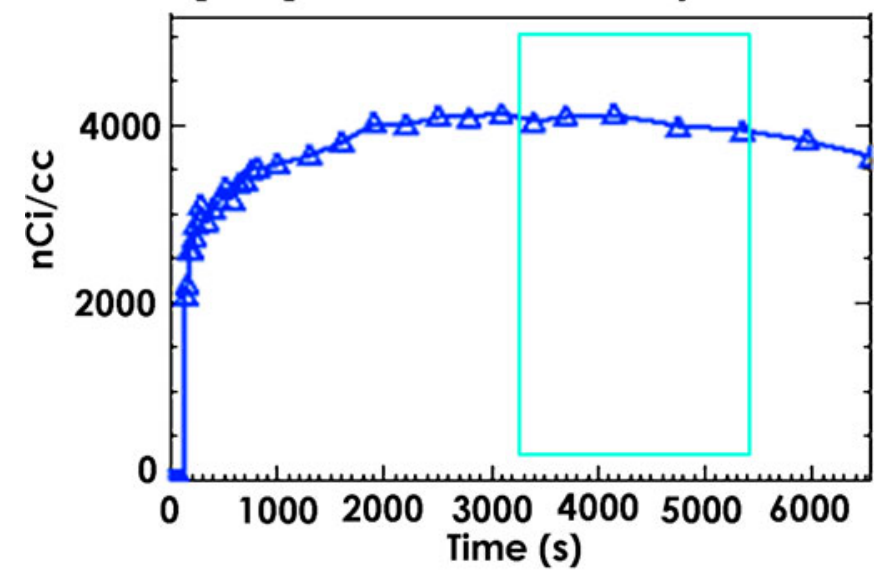

[13N]NH4+ Time-Activity Curve

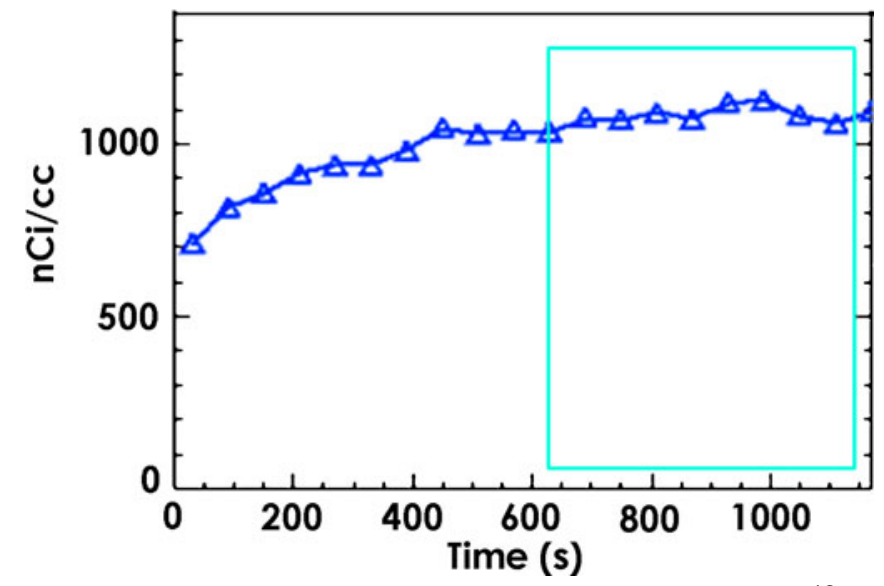

Fig. 2. Time-activity curve (TAC) of both radiotracers, ${ }^{18} \mathrm{~F}$ FDG and ${ }^{13} \mathrm{~N}-\mathrm{NH}_{4}{ }^{+}$. Two single dynamic studies were acquired, one for each radiotracer, in order to ensure that the radiotracers activity in the rat brain do not change along time when applying SDTT.

\section{Results and Discussion}

\section{Phantom Experiments}

Table 1 presents the theoretical and experimental concentrations for ${ }^{18} \mathrm{~F}-\mathrm{FDG}$ and ${ }^{13} \mathrm{~N}-\mathrm{NH}_{4}{ }^{+}$(IM and SM results), for SSRB (span, 3 and ring difference, 31), total acquisition time of $30 \mathrm{~min}$ and frame duration of $60 \mathrm{~s}$. No significant differences were observed for other rebinning algorithms, total acquisition times and frame durations. As it can be observed, the resulting concentrations after SDTT are quite similar to the theoretical ones, the true concentration of each one is recovered. There are no significant differences between the IM and SM methods. The SDTT method provides the least accurate results when the two radiotracers are in similar concentrations, and a negative bias of about $7 \%$ can be observed for ${ }^{18} \mathrm{~F}-\mathrm{FDG}$, and the opposite for ${ }^{13} \mathrm{~N}_{-} \mathrm{NH}_{4}{ }^{+}$. When one of the radiotracers is in the majority, this bias also exists but presents a lower magnitude in the resulting images.
Fig. 3 shows that the SNR depends on the rebinning algorithms and the total acquisition times. In contrast, it also shows that the SNR was independent of the frame duration.

Influence of Rebinning Algorithms Fig. 4 illustrates, in relation to the rebinning algorithms, the results shown in Fig. 3, which ROIs correspond to each Eppendorf tube and also the TACs of ${ }^{18} \mathrm{~F}-\mathrm{FDG},{ }^{13} \mathrm{~N}_{-} \mathrm{NH}_{4}{ }^{+}$and of the dual isotope. From both figures, it can be observed that different rebinning algorithms applied to the SDTT give different results, with the image quality depending on the rebinning algorithm. For example, using an SSRB sinogram with a span of 3 and ring difference of 31, an image with higher SNR is obtained (i.e. with less noise) than using a 3D sinogram and FORE reconstruction. Applying SDTT after an acquisition time of $30 \mathrm{~min}$, the ${ }^{18} \mathrm{~F}$-FDG SNR of the images obtained using SSRB is between 45 and 50, regardless of the span and ring difference. In contrast, using a $3 \mathrm{D}$ sinogram and FORE in the reconstruction, the SNR value is approximately 15 (Fig. 3).

This result can be explained by the fact that when using a 3D sinogram and a FORE reconstruction, each voxel presents a lower number of counts, and therefore a worse SNR, given the Poisson nature of noise in PET imaging. In contrast, if an SSRB sinogram is used and an adequate span and ring difference is applied, higher SNR values are achieved because of the better per-voxel counting statistics. In addition, the worse SNR results appear in the FORE image due to an artefact which is clearly visible in Fig. 4 (lower right). This artefact arises from the fact that the FORE algorithm is not exact and starts degrading the resolution of the images when the acceptance angle is over $25^{\circ}\left(26.85^{\circ}\right.$ in our case). The influence of different rebinning algorithms on PET imaging have already been studied, where different exact and approximate rebinning algorithms for $2 \mathrm{D}$ and 3D PET data were evaluated and compared [12, 14].

Table 2 shows the coefficient of variation of all the ROIs in the ${ }^{18} \mathrm{~F}-\mathrm{FDG}$ and ${ }^{13} \mathrm{~N}-\mathrm{NH}_{4}{ }^{+}$images for different rebinning algorithms. As can be observed, the coefficients of variation do not vary significantly with the different rebinning algorithms. This can be explained by the fact that artefacts arising from the FORE method would add up variance in the background, thus lowering the SNR for that particular image without affecting the $\mathrm{CV}$ calculation.

Influence of Total Acquisition Time Fig. 5 shows the resulting ${ }^{18} \mathrm{~F}-\mathrm{FDG}$ and ${ }^{13} \mathrm{~N}_{-} \mathrm{NH}_{4}^{+}$images using the IM. When the total acquisition time is over $20 \mathrm{~min}$, the resulting images are of a similar quality to the $30 \mathrm{~min}$ static image, but for lower total acquisition times (e.g. 5 or $10 \mathrm{~min}$ ) the images are much noisier.

The SNR of the initial image $\left({ }^{18} \mathrm{~F}-\mathrm{FDG}\right.$ and ${ }^{13} \mathrm{~N}_{-} \mathrm{NH}_{4}{ }^{+}$ combined; SSRB sinogram with a span of 3 and a ring difference of 31 ; total acquisition time of $30 \mathrm{~min}$; and a frame duration of $60 \mathrm{~s}$ ) was 43.65 and, after applying the 
Table 1. Accuracy of SDTT. Percentage of ${ }^{18} \mathrm{~F}-\mathrm{FDG}$ and ${ }^{13} \mathrm{~N}_{-} \mathrm{NH}_{4}{ }^{+}$concentrations of all the ROIs; the theoretical values, IM and SM results

\begin{tabular}{|c|c|c|c|c|c|c|c|c|c|c|}
\hline \multirow[t]{2}{*}{ ROI } & \multicolumn{2}{|c|}{ Theorethical values } & \multicolumn{2}{|c|}{ IM results } & \multicolumn{2}{|l|}{ Bias } & \multicolumn{2}{|c|}{ SM results } & \multicolumn{2}{|l|}{ Bias } \\
\hline & $\%$ FDG & $\% \mathrm{NH} 3$ & $\%$ FDG & $\% \mathrm{NH} 3$ & $\%$ FDG & $\% \mathrm{NH} 3$ & $\%$ FDG & $\% \mathrm{NH} 3$ & $\%$ FDG & $\% \mathrm{NH} 3$ \\
\hline 1 & 90.667 & 9.333 & 91.787 & 8.213 & 1.120 & -1.120 & 92.555 & 7.445 & 1.888 & -1.888 \\
\hline 2 & 100.000 & 0.000 & 101.057 & -1.057 & 1.057 & -1.057 & 101.913 & -1.913 & 1.913 & -1.913 \\
\hline 3 & 74.453 & 25.547 & 72.491 & 27.509 & -1.962 & 1.962 & 73.111 & 26.889 & -1.341 & 1.341 \\
\hline 4 & 56.432 & 43.568 & 48.973 & 51.027 & -7.458 & 7.458 & 49.426 & 50.574 & -7.006 & 7.006 \\
\hline 5 & 32.692 & 67.308 & 27.782 & 72.218 & -4.910 & 4.910 & 28.088 & 71.912 & -4.605 & 4.605 \\
\hline 6 & 0.000 & 100.000 & -0.089 & 100.089 & -0.089 & 0.089 & 0.064 & 99.936 & 0.064 & -0.064 \\
\hline
\end{tabular}

Bias is the percentage of the difference between the theoretical values and the experimental results. Experimental settings SSRB sinogram with a span of 3 and ring difference of 31 . Total acquisition time, $30 \mathrm{~min}$. Frame duration, $60 \mathrm{~s}$

SDTT with the IM, the resulting ${ }^{18} \mathrm{~F}-\mathrm{FDG}$ and ${ }^{13} \mathrm{~N}_{-} \mathrm{NH}_{4}{ }^{+}$ images presented SNR values of 50.32 and 39.00, respectively, and when using the sinogram method 50.21 and 38.97 , respectively. When the acquisition time is $30 \mathrm{~min}$, both methods discriminate ${ }^{18} \mathrm{~F}-\mathrm{FDG}$ and ${ }^{13} \mathrm{~N}_{-} \mathrm{NH}_{4}{ }^{+}$images with similar SNR values compared to the initial dynamic image. ${ }^{18} \mathrm{~F}$-FDG images present less noise than the initial dynamic image. In contrast, the ${ }^{13} \mathrm{~N}_{-} \mathrm{NH}_{4}{ }^{+}$images are slightly noisier; for example, applying SDTT using SSRB with a span of 3 and ring difference of 31 , when the total acquisition time is between 20 and $30 \mathrm{~min}$, the SNR of ${ }^{18} \mathrm{~F}$ FDG images is approximately 50 . On the other hand, when the total acquisition time is $10 \mathrm{~min}$ the ${ }^{18} \mathrm{~F}$-FDG images present an SNR of 12 (Fig. 3).

Table 3 illustrates the CV of all the ROIs in the ${ }^{18} \mathrm{~F}-\mathrm{FDG}$ and ${ }^{13} \mathrm{~N}_{-} \mathrm{NH}_{4}{ }^{+}$images for different total acquisition times.

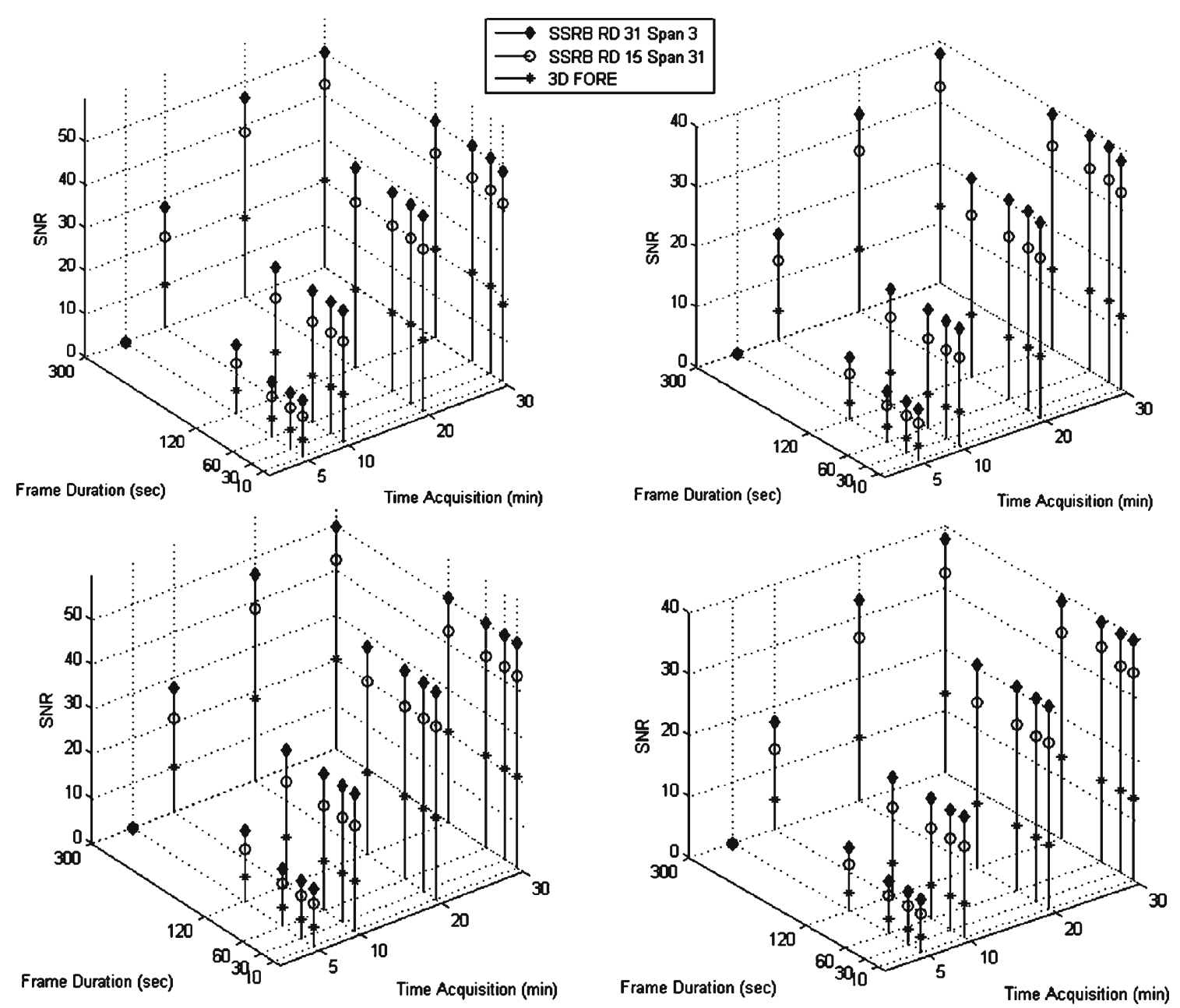

Fig. 3. Signal to noise ratio (SNR) values of the ${ }^{18} \mathrm{~F}-\mathrm{FDG}$ (right) and ${ }^{13} \mathrm{~N}_{-} \mathrm{NH}_{4}{ }^{+}$(left) images after applying the Sinogram Method (upper row) and the Image Method, using different rebinning algorithms, total time acquisition (5, 10, 20, and $30 \mathrm{~min})$, and frame duration $(10,30,60,120,300 \mathrm{~s})$. 


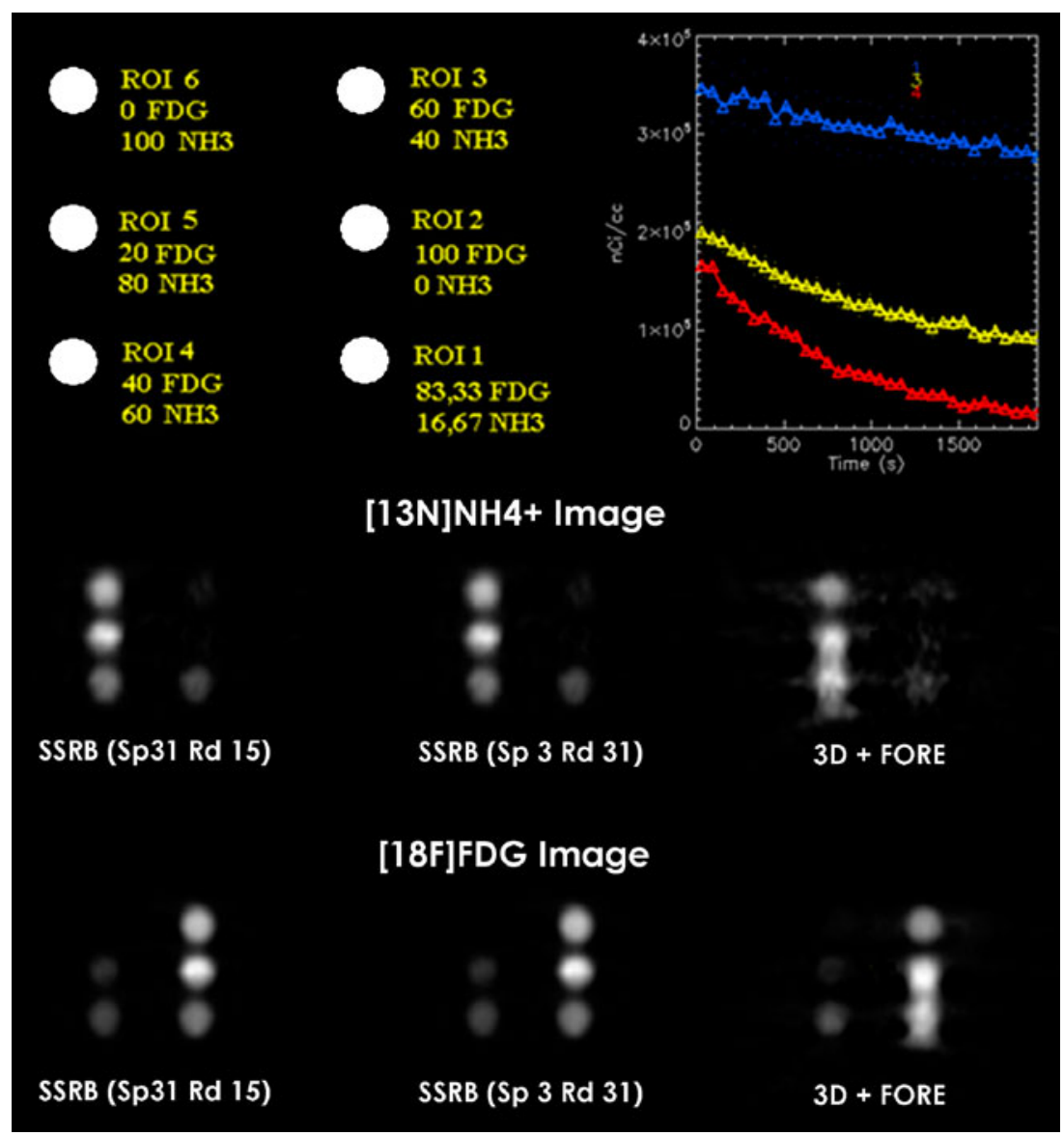

Fig. 4. Top relative volumes of ${ }^{18} \mathrm{~F}-\mathrm{FDG}$ and ${ }^{13} \mathrm{~N}-\mathrm{NH}_{4}{ }^{+}$introduced on each Eppendorf tube (left). Time-activity curves (TACs) of ${ }^{18} \mathrm{~F}-\mathrm{FDG}$ (blue ROI 2), for ${ }^{13} \mathrm{~N}-\mathrm{NH}_{4}{ }^{+}$(red ROI 6) and for dual-isotope (yellow ROI 4; right). Down ${ }^{13} \mathrm{~N}-\mathrm{NH}_{4}{ }^{+}$(upper row) and ${ }^{18} \mathrm{~F}-$ FDG images for different rebinning algorithms (left to right) SSRB with a span of 31 and a ring difference of 15; SSRB with a span of 3 and ring difference of 31; 3D Sinogram and FORE on the reconstruction. Experimental settings: total time acquisition of $30 \mathrm{~min}$ and frame duration of $60 \mathrm{~s}$. As it can be observed, using a SSRB sinogram with a span of 3 and ring difference of 31 , the resulted images present less noise than for, e.g. using a 3D sinogram and a Fourier rebinning reconstruction.

As expected, a longer acquisition time gives a lower $\mathrm{CV}$ and therefore less noise in the resulting images.

Both IM and SM methods discriminate ${ }^{18}$ F-FDG and ${ }^{13} \mathrm{~N}_{-} \mathrm{NH}_{4}{ }^{+}$images with similar quality in comparison to the initial dynamic image (Table 1$).{ }^{18}$ F-FDG images present less noise than the initial dynamic image, as the ${ }^{18}$ F-FDG signal hardly varies over the total acquisition time and therefore the noise is cancelled out when fitting the data. In contrast, ${ }^{13} \mathrm{~N}-\mathrm{NH}_{4}{ }^{+}$images are slightly noisier due to their different initial activity concentration, since the ${ }^{18}$ F-FDG activity concentration was approximately double that of ${ }^{13} \mathrm{~N}_{-} \mathrm{NH}_{4}{ }^{+}$. Subsequently, there are fewer counts on the ${ }^{13} \mathrm{~N}-\mathrm{NH}_{4}{ }^{+}$images, which also imply a lower SNR.

The main factor influencing the performance of the SDTT is the per-voxel counting statistics. Current scanner sensitivities are higher by comparison with those when SDTT was originally proposed [4, 15, 16]. Higher sensitivity

Table 2. Coefficient of variation of all the ROIs in the ${ }^{18} \mathrm{~F}-\mathrm{FDG}$ and ${ }^{13} \mathrm{~N}_{-} \mathrm{NH}_{4}{ }^{+}$images for different rebinning algorithms

\begin{tabular}{|c|c|c|c|c|c|c|}
\hline \multirow[t]{2}{*}{ ROI } & \multicolumn{3}{|l|}{ FDG } & \multicolumn{3}{|l|}{ NH3 } \\
\hline & $\mathrm{SSRB} s=31 ; \mathrm{RD}=15$ & $\mathrm{SSRB} s=3 ; \mathrm{RD}=31$ & FORE & $\mathrm{SSRB} s=31 ; \mathrm{RD}=15$ & $\mathrm{SSRB} s=3 ; \mathrm{RD}=31$ & FORE \\
\hline 1 & 0.841 & 0.838 & 0.722 & 1.208 & 1.194 & 0.873 \\
\hline 2 & 0.841 & 0.837 & 0.769 & - & - & - \\
\hline 3 & 0.859 & 0.855 & 0.734 & 0.853 & 0.848 & 1.057 \\
\hline 4 & 0.693 & 0.687 & 0.697 & 0.662 & 0.659 & 0.604 \\
\hline 5 & 0.701 & 0.697 & 0.663 & 0.674 & 0.670 & 0.645 \\
\hline 6 & - & - & - & 0.514 & 0.512 & 0.473 \\
\hline
\end{tabular}

Experimental settings total acquisition time, $30 \mathrm{~min}$; frame duration, $60 \mathrm{~s}$ 


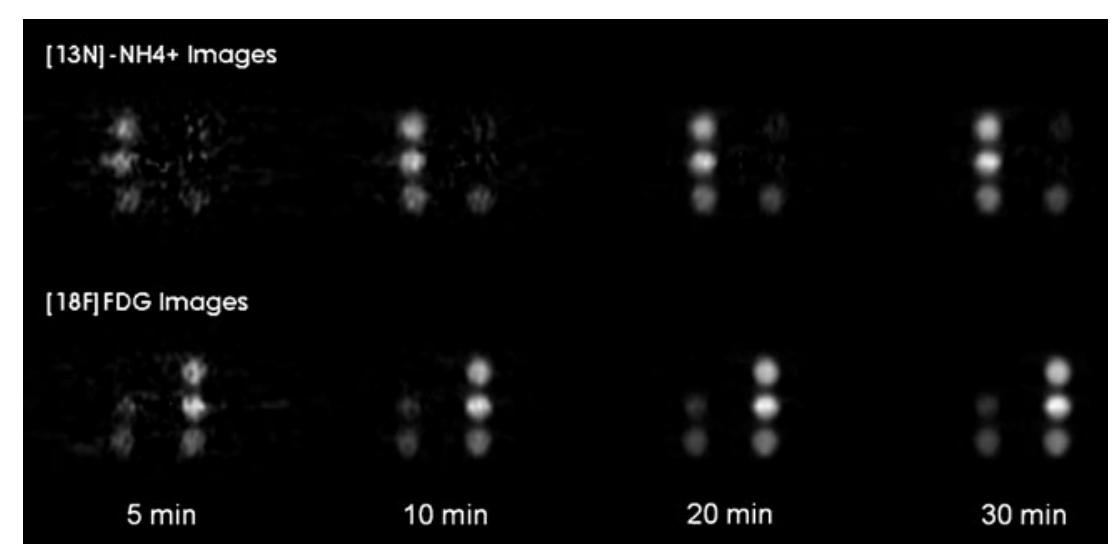

Fig. 5. ${ }^{13} \mathrm{~N}_{-} \mathrm{NH}_{4}{ }^{+}$(upper row) and ${ }^{18} \mathrm{~F}-\mathrm{FDG}$ Images for different total acquisition time: 5, 10, 20, and 30 min. Experimental settings SSRB sinogram with a span of 3 and ring difference of 31 and frame duration of $60 \mathrm{~s}$. As can be observed, lower total time acquisition images are noisier.

means better per-voxel statistics and current PET scanners allow the use of this technique. However, the increased sensitivity is partially offset by the improvement in spatial resolution. Modern PET scanners also offer better spatial resolution, enabling smaller voxel sizes, and therefore yielding lesser counts per voxel at a certain activity concentration. Thus, even with higher sensitivities the better per-voxel statistics achieved are also dependent on the spatial resolution of the scanner and, therefore, on voxel size [16-18]. In this study, for an acquisition of 20 and $30 \mathrm{~min}$, approximately $9-13$ and $13-19 \times 10^{6}$ prompts were achieved.

\section{In vivo Experiments}

Kinetic Evaluation Fig. 2 shows the TAC in the rat brain of the two radiotracers, ${ }^{18} \mathrm{~F}-\mathrm{FDG}$ and ${ }^{13} \mathrm{~N}_{-} \mathrm{NH}_{4}{ }^{+}$. Between approximately 30 and $100 \mathrm{~min}(\sim 2,000-6,000 \mathrm{~s})$ of image acquisition, ${ }^{18} \mathrm{~F}-\mathrm{FDG}$ does not change significantly over time (Fig. 2- ${ }^{18}$ F-FDG TAC). Moreover, from approximately $10 \mathrm{~min}(600 \mathrm{~s})$ of image acquisition, ${ }^{13} \mathrm{~N}^{-\mathrm{NH}_{4}}{ }^{+}$also does not vary appreciably with time (Fig. $2-{ }^{13} \mathrm{~N}_{-} \mathrm{NH}_{4}{ }^{+}$ TAC). From Fig. 2 and the protocol used in the in vivo experiments, it was determined that SDTT can be applied approximately $10 \mathrm{~min}$ after ${ }^{13} \mathrm{~N}_{-} \mathrm{NH}_{4}{ }^{+}$injection (5 min after the starting of image acquisition in our case) and during the following $30 \mathrm{~min}$ since there is no significant variation in radiotracer concentration. In all the in vivo experiments, SDTT was applied after $5 \mathrm{~min}$ of image acquisition and for $30 \mathrm{~min}$.

Experiment 1: The aim of the first in vivo experiment was to test the method in a situation where the resulting ${ }^{13} \mathrm{~N}-\mathrm{NH}_{4}{ }^{+}$and ${ }^{18} \mathrm{~F}$ FDG images should be different: a rat with transient ischemia was studied at $18 \mathrm{~h}$ post-reperfusion. At this time, the ${ }^{18}$ F-FDG uptake is known to be reduced in the region of the ischemia but the blood flow could persist in the infarcted tissue despite the massive cell death [10]. In the ${ }^{18} \mathrm{~F}-\mathrm{FDG}$ image, the low uptake of ${ }^{18} \mathrm{~F}-\mathrm{FDG}$ in the infarcted region is clearly visible (Fig. 6a), as confirmed after brain slice staining with the 2,3,5-triphenyltetrazolium chloride method (Fig. 7). This region maintains a nearly normal blood perfusion, as reflected in the image of ${ }^{13} \mathrm{~N}_{-} \mathrm{NH}_{4}{ }^{+}$(Fig. 6b). However, the asymmetry in the soft tissue of the head is clearly visible, reflecting a reduced blood flow in the ipsilateral face muscles as a consequence of the method used to produce the ischemia. In this method, the

Table 3. $\mathrm{CV}$ of all the ROIs in the ${ }^{18} \mathrm{~F}-\mathrm{FDG}$ and ${ }^{13} \mathrm{~N}-\mathrm{NH}_{4}{ }^{+}$images for different total acquisition times

\begin{tabular}{|c|c|c|c|c|c|c|c|c|}
\hline \multirow[t]{2}{*}{ ROI } & \multicolumn{4}{|l|}{ FDG } & \multicolumn{4}{|l|}{ NH3 } \\
\hline & $5 \mathrm{~min}$ & $10 \mathrm{~min}$ & $20 \mathrm{~min}$ & $30 \mathrm{~min}$ & $5 \mathrm{~min}$ & $10 \mathrm{~min}$ & $20 \mathrm{~min}$ & $30 \mathrm{~min}$ \\
\hline 1 & 0.929 & 0.845 & 0.838 & 0.838 & 5.330 & 1.945 & 1.401 & 1.194 \\
\hline 2 & 0.958 & 0.858 & 0.842 & 0.837 & - & & - & - \\
\hline 3 & 1.062 & 0.877 & 0.859 & 0.855 & 1.711 & 1.035 & 0.877 & 0.848 \\
\hline 4 & 1.121 & 0.757 & 0.694 & 0.687 & 1.063 & 0.779 & 0.674 & 0.659 \\
\hline 5 & 1.523 & 0.831 & 0.709 & 0.697 & 0.862 & 0.700 & 0.681 & 0.670 \\
\hline 6 & - & - & - & - & 0.612 & 0.517 & 0.511 & 0.512 \\
\hline
\end{tabular}

Experimental settings: SSRB sinogram with a span of 3, ring difference of 31 and a frame duration of $60 \mathrm{~s}$ 
Experiment 1
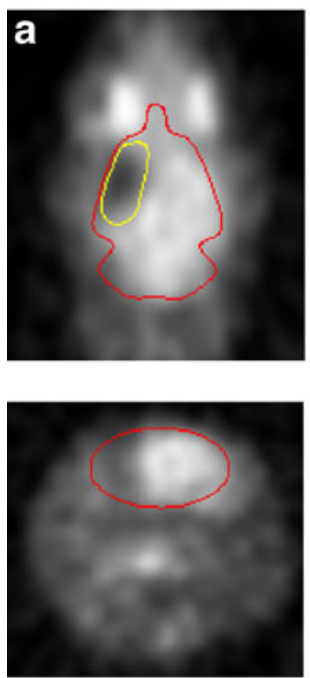

${ }^{18} \mathrm{FFDG}$
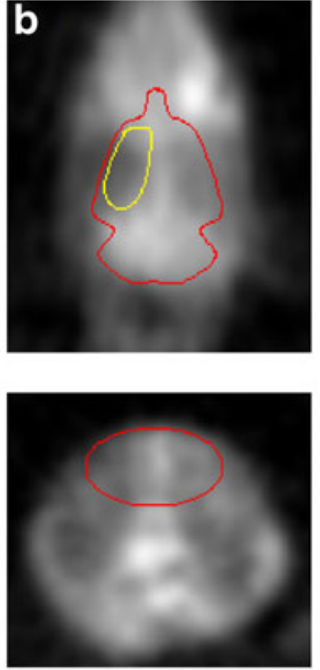

${ }^{13} \mathrm{~N}-\mathrm{NH}_{4}{ }^{+}$
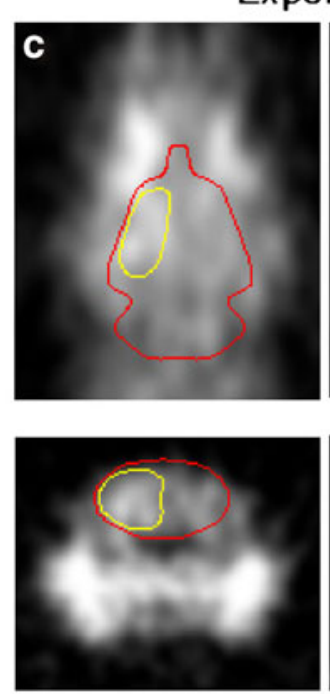

${ }^{18} \mathrm{~F}-\mathrm{FDG}$

\section{Experiment 2}
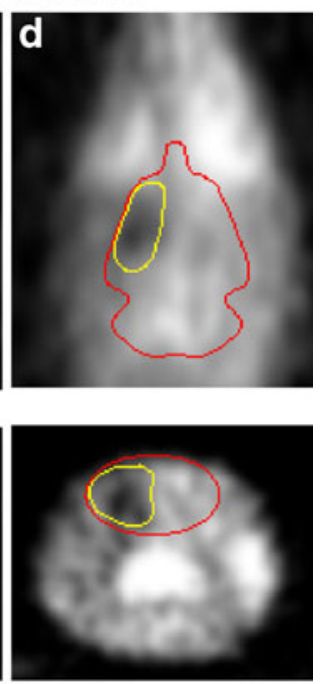

${ }^{13} \mathrm{~N}-\mathrm{NH}_{4}{ }^{+}$

\section{Experiment 3 (Control)}
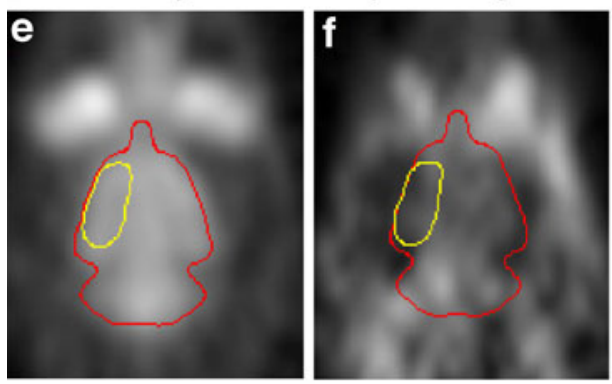

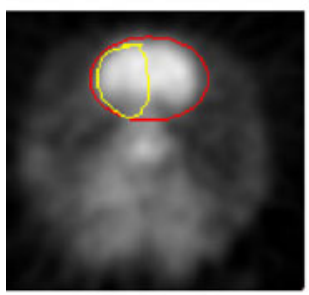

${ }^{18} \mathrm{~F}-\mathrm{FDG}$

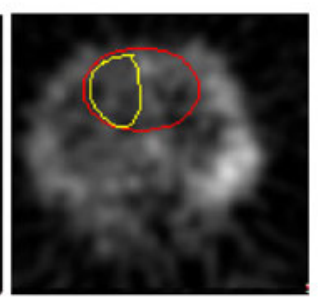

${ }^{13} \mathrm{~N}-\mathrm{NH}_{4}^{+}$

Fig. 6. In vivo experiments ${ }^{13} \mathrm{~N}-\mathrm{NH}_{4}{ }^{+}$and ${ }^{18} \mathrm{~F}-\mathrm{FDG}$ resultant images after applying SDTT; a low uptake of ${ }^{18} \mathrm{~F}-\mathrm{FDG}$ in the ischemic territory (outlined in yellow) indicating tissue necrosis; b nearly symmetric blood perfusion in the brain despite the infarct indicating a successful reperfusion in the same brain area. The soft tissue of the head presents asymmetric blood flow, caused by the experimental method in which the external carotid artery was closed and used to introduce de occlusion filament; c normal ${ }^{18} \mathrm{~F}-\mathrm{FDG}$ with a slightly elevated uptake in the ischemic territory; $\mathbf{d}$ the same animal present a severe asymmetry in cerebral perfusion secondary to the middle cerebral artery occlusion; e normal ${ }^{18} \mathrm{~F}-\mathrm{FDG}$ with no detectable asymmetry; f symmetric perfusion in the brain.

external carotid artery remains irreversibly closed after using it to introduce the occlusion filament into the internal carotid artery to occlude the middle cerebral artery. The decreased blood flow in the soft tissues observed here does not cause damage to these regions. To our knowledge, this is the first time that successful in vivo dual-isotope PET imaging has been reported.
Table 4 shows the ratio between the uptake of the affected vs. contralateral hemispheres of the brain in the resulting ${ }^{18} \mathrm{~F}$-FDG and ${ }^{13} \mathrm{~N}_{-} \mathrm{NH}_{4}{ }^{+}$images. These values are in agreement with previously reported data employing an identical animal model, but using the radiotracers separately [10]. As expected, in the ${ }^{18} \mathrm{~F}-\mathrm{FDG}$ image the affected hemisphere (infarcted tissue) presents a lower uptake and consequently the ratio between affected vs contralateral hemispheres is also low (0.651). In the ${ }^{13} \mathrm{~N}_{-} \mathrm{NH}_{4}{ }^{+}$image, the affected/contralateral ratio is 0.896 , meaning that the blood 


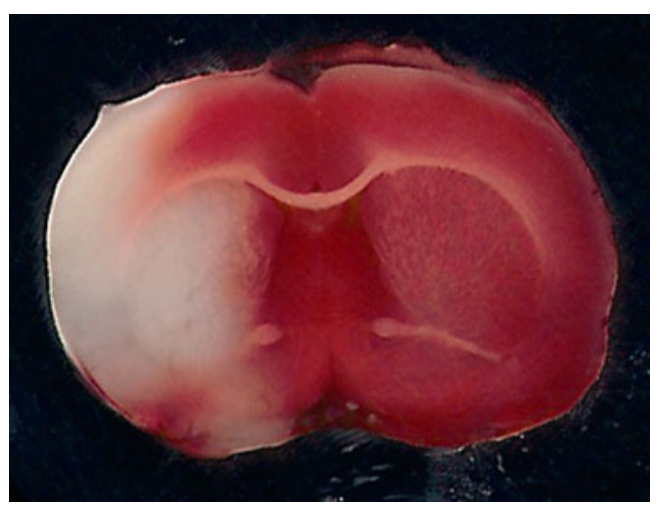

Fig. 7. Representative brain slice stained with 2,3,5-Triphenyl tetrazolium chloride method. Pale areas correspond to infarcted tissue which is visible at the left hand side.

flow persists in the infarcted tissue despite the massive cell death.

Experiment 2: The aim of the second in vivo experiment was to test the method in the contrary situation. In this case, ${ }^{18} \mathrm{~F}$-FDG was injected $1 \mathrm{~h}$ before inducing the ischemia to permit normal uptake of this tracer, and the ${ }^{13} \mathrm{~N}-\mathrm{NH}_{4}{ }^{+}$was injected immediately after the ischemic procedure. This approach allowed us to obtain a nearly symmetric ${ }^{18}$ F-FDG image corresponding to normal brain uptake (Fig. 6c) and an asymmetric ${ }^{13} \mathrm{~N}_{-} \mathrm{NH}_{4}{ }^{+}$image corresponding to the reduction in the cerebral blood flow in the region of the occluded middle cerebral artery (Fig. 6d).

As it can be observed in Table 4, the affected tissue (right hemisphere) in the resulting ${ }^{13} \mathrm{~N}_{-} \mathrm{NH}_{4}{ }^{+}$image, presents an affected/contralateral ratio of 0.634 signifying that the cerebral blood flow is reduced.

As regards the resulting ${ }^{18} \mathrm{~F}-\mathrm{FDG}$ image, the right/left ratio obtained is higher than expected (1.254). This can be attributable to the manipulations of the carotid territory during surgery which might have triggered an adaptive process of the brain parenchyma. Known mechanisms of adaptation to reduced blood flow include transient vasodilatation and increased glycolytic activity. Both these mechanisms would lead to an increased uptake of ${ }^{18}$ F-FDG. Similarly, a glycolytic burst in the ischemic parenchyma in the initial minutes following the introduction of the occlusion monofilament could have occurred. This phenomenon would be related to the excitotoxicity or spreading depression well known to occur in the region of the middle cerebral artery during early stages of cerebral ischemia. However, the actual mechanism of this increased uptake is as yet unknown, since studies using ${ }^{18}$ F-FDG that take the same approach as we adopted in our work have not been reported. In any event, the explanation of this phenomenon is beyond the scope of the present study, and future studies could be useful in order to shed light on the initial changes that occur in the glucose metabolism induced by the ischemia.

Experiment 3: The aim of the third in vivo experiment was to test the method in a situation where ${ }^{18} \mathrm{~F}$ FDG and ${ }^{13} \mathrm{~N}_{-} \mathrm{NH}_{4}{ }^{+}$images (Fig. 6e and f) present a normal brain uptake and a symmetric blood flow (control experiment). In this case, ${ }^{18} \mathrm{~F}$-FDG was injected into the rat before surgery, and therefore a normal symmetric ${ }^{18}$ F-FDG image was obtained as expected. After surgery, ${ }^{13} \mathrm{~N}_{-} \mathrm{NH}_{4}{ }^{+}$was injected into the sham-operated rat. Since there was not MCA occlusion, a symmetrical ${ }^{13} \mathrm{~N}-\mathrm{NH}_{4}{ }^{+}$image was obtained showing the expected normal blood flow.

Table 4 shows the ratio between right and left brain hemispheres in the resulting ${ }^{18} \mathrm{~F}-\mathrm{FDG}$ and ${ }^{13} \mathrm{~N}_{-} \mathrm{NH}_{4}{ }^{+}$ images. The results were as expected, a symmetric ${ }^{18} \mathrm{~F}$ FDG and ${ }^{13} \mathrm{~N}_{-} \mathrm{NH}_{4}{ }^{+}$images meaning normal brain uptake and symmetric cerebral blood flow (right/left ratio of approximately 1 ).

\section{Conclusion}

This study demonstrates that simultaneous dual-tracer imaging is practicable in rodent scanners and accuracy depends on the experimental settings. The current generation of PET scanners is more sensitive and provides better image resolution than when this technique was originally proposed [4]. The procedure has not been routinely applied since that date because it could lead to the presence of increased noise in the images. However, this study has shown that SDTT is applicable when total acquisition times are over $20 \mathrm{~min}$ and radiotracer concentrations do not vary during this period. Given that no significant differences were observed between the image and sinogram methods, the former is preferred because it is faster and simpler to apply. Using the SSRB with a span of 3 and a ring difference of 31 , and acquiring for at least $20 \mathrm{~min}$, independently of the frame duration, this technique does distinguish between the two radiotracer images with similar SNR values to the initial dynamic image. It may also be concluded that SDTT does not enhance noise significantly in

Table 4. Uptake ratios of the right vs. left brain hemispheres in the resulting ${ }^{18} \mathrm{~F}$-FDG and ${ }^{13} \mathrm{~N}-\mathrm{NH}_{4}{ }^{+}$images (affected and contralateral hemispheres for experiments 1 and 2)

\begin{tabular}{lll}
\hline Experiment & Image & Right/left ratio \\
\hline 1 Infarct & FDG & 0.651 \\
& NH3 & 0.896 \\
2 Ischemia & FDG & 1.254 \\
3 Control & NH3 & 0.634 \\
& FDG & 1.004 \\
& NH3 & 0.921 \\
\hline
\end{tabular}


the resulting images if the experimental settings are correctly chosen: total acquisition time and rebinning algorithm.

The SDTT procedure presented here does have its limitations; SDTT assumes that tracer concentrations do not change during the acquisition; therefore, SDTT is not applicable to kinetic studies. It can only be applied in static studies, when it can be ensured that both radiotracer concentrations do not vary, and the acquisition time of the experiment is at least $20 \mathrm{~min}$. Shorter acquisition times will generate noisier images. However, these restrictions are similar to those usually found with diagnostic PET protocols using static imaging with ${ }^{18} \mathrm{~F}-\mathrm{FDG}$ or ${ }^{13} \mathrm{~N}-\mathrm{NH}_{4}{ }^{+}$.

The SDTT was applied using a rodent system. To apply SDTT using human scanners, the number of coincidence events on human imaging voxel must be similar to that achieved on a typical rodent imaging "voxel". Apart from the difference between human and rodent scanners in terms of spatial resolution and sensitivity, there is another limitation in human studies; the injected radiotracer concentration. The injected radiotracer concentration used in this work is higher than is usually used in human studies. Consequently, the pervoxel coincidence events achieved in this work were higher than those currently achievable in human studies. Reasonable per-voxel counting statistics on human scanners and, consequently, a reasonable SDTT performance, would require an increase in scanner sensitivity, an increase in voxel size or increase of total scanning time. However, this last option is limited to the kinetics of the employed radiotracers.

The SDTT was shown to be feasible in a rodent model of transient cerebral ischemia, obtaining results for both ${ }^{18} \mathrm{~F}$ FDG and ${ }^{13} \mathrm{~N}_{-} \mathrm{NH}_{4}{ }^{+}$very similar to those obtained in single tracer studies in the same animal model [10]. The SDTT will be applied in future studies that focus on the relationship between the blood flow and other pathological processes implicated in ischemic damage.

Results from this study are encouraging and prove that this technique is practicable in rodent scanners. Future work will also focus on evaluating the role of iterative reconstruction algorithms, such as the OSEM algorithm.

Acknowledgements. This work was partially supported by the Ministerio de Industria, Turismo y Comercio (Cenit-Ingenio Program: CDTEAM project).

Francisca P. Figueiras has a Ph.D fellowship from the Fundação para a Ciência e a Tecnologia , POCI 2010, FSE SFRH/BD/38341/2007, and the European Community's Seventh Framework Program (FP7/2007-2013), HEALTH F2 2008200728.

Disclosure/Conflict of Interest. The authors declare that they have no proprietary, financial, professional or other personal interest of any nature or kind in any product, service and/or company that could be construed as influencing the position presented in, or the review of, this manuscript.

\section{References}

1. Kiat H, Germano G, Friedman J, Van Train K, Silagan G, Wang FP, Maddahi J, Berman D (1994) Comparative feasibility of separate or simultaneous rest thallium-201/stress technetium-99m-sestamibi dualisotope myocardial perfusion SPECT. J Nucl Med 35(4):542-548

2. Unlu M, Gunaydin S, Ilgin N, Inanir S, Gokcora N, Gokgoz L (1993) Dual isotope myocardial perfusion SPECT in the detection of coronary artery disease: comparison of separate and simultaneous acquisition protocols. J Nucl Biol Med 37(4):233-237

3. Wu YW, Huang PJ, Lee CM, Ho YL, Lin LC, Wang TD, Wang SS, Chen TH, Yen RF (2005) Assessment of myocardial viability using F18 fluorodeoxyglucose/Tc-99m sestamibi dual-isotope simultaneous acquisition SPECT: comparison with Tl-201 stress-reinjection SPECT. J Nucl Cardiol 12(4):451-459

4. Huang SC, Carson RE, Hoffman EJ, Kuhl DE, Phelps ME (1982) An investigation of a double-tracer technique for positron computerized tomography. J Nucl Med 23(9):816-822

5. Verhaeghe J, D'Asseler Y, Staelens S, Lemahieu I (2005) Noise properties of simultaneous dual tracer PET imaging. IEEE Nuclear Science Symposium, San Juan

6. Black NF, McJames S, Rust TC, Kadrmas DJ (2008) Evaluation of rapid dual-tracer (62)Cu-PTSM + (62)Cu-ATSM PET in dogs with spontaneously occurring tumors. Phys Med Biol 53(1):217-232

7. Ikoma Y, Toyama H, Suhara T (2004) Simultaneous quantification of two brain functions with dual tracer injection in PET dynamic study. In: Iida H, Shah NJ, Hayashi T, Watabe H (eds) Quantitation in biomedical imaging with PET and MRI. Quantitation in PET and image processing, 1st edn. Elsevier, Amsterdam, The Netherlands, pp 74-78

8. Rust TC, Kadrmas DJ (2006) Rapid dual-tracer PTSM+ATSM PET imaging of tumour blood flow and hypoxia: a simulation study. Phys Med Biol 51(1):61-75

9. Wilson JW, Turkington TG, Colsher JG, Borges-Neto S, Reinman RE, Coleman E (2004) Optimizing sequencial dual tracer PET studies using combined 2D/3D Imaging Protocol. in IEEE Nuclear Science Symposium. Rome (Italy)

10. Martín A, Rojas S, Pareto D, Santalucia T, Millán O, Abasolo I, Gómez V, Llop J, Gispert JD, Falcon C, Bargalló N, Planas AM (2008) Depressed glucose consumption at reperfusion following brain ischemia does not correlate with mitochondrial dysfunction and development of infarction: an in vivo positron emission tomography study. Curr Neurovasc Res 6(2):82-88

11. Daube-Witherspoon ME, Muehllehner G (1987) Treatment of axial data in three-dimensional PET. J Nucl Med 28(11):1717-1724

12. Defrise M, Kinahan PE, Townsend DW, Michel C, Sibomana M, Newport DF (1997) Exact and approximate rebinning algorithms for 3D PET data. IEEE Trans Med Imaging 16(2):145-158

13. Martin A, Rojas S, Chamorro A, Falcon C, Bargallo N, Planas AM (2006) Why does acute hyperglycemia worsen the outcome of transient focal cerebral ischemia? Role of corticosteroids, inflammation, and protein O-glycosylation. Stroke 37(5):1288-1295

14. Bouallegue FB, Crouzet JF, Comtat C, Fourcade M, Mohammadi B, Mariano-Goulart D (2007) Exact and approximate Fourier rebinning algorithms for the solution of the data truncation problem in 3-D PET. IEEE Trans Med Imaging 26(7):1001-1009

15. Valk PE, Dale L, Bailey D, Townsend DW, Maisey MN (2004) Positron emission tomography: basic science and clinical practice. Springer, London

16. Vaquero JJ, Desco M (2005) Technical limitations of the positron emission tomography (PET) for small laboratory animals. Rev Esp Med Nucl 24(5):334-347

17. Jagoda EM, Vaquero JJ, Seidel J, Green MV, Eckelman WC (2004) Experiment assessment of mass effects in the rat: implications for small animal PET imaging. Nucl Med Biol 31(6):771-779

18. trother SC, Casey ME, Hoffman EJ (1990) Measuring PET scanner sensitivity: relating count rates to image signal-to-noise ratios using Noise Equivalent Counts, in IEEE T Nucl Sci 